\title{
Signs of gas flows in the variable Balmer line profiles of the Seyfert galaxy nuclei NGC 3227 and NGC 7469
}

\author{
I. I. Pronik
}

Crimean Astrophysical Observatory and Isaac Newton Institute of Chile, Crimean Branch, 98409 Nauchny, Crimea, Ukraine e-mail: ipronik@crao.crimea.ua

Received 2 December 2007 / Accepted 10 October 2008

\section{ABSTRACT}

\begin{abstract}
The data on the variable Balmer line profiles obtained by us for the Seyfert galaxy NGC 3227 and NGC 7469 nuclei and published separately during 1975-2006, were combined with the data compiled from literature, and reviewed in this paper. We hypothesize that the profile variations are connected with three independent regions of different physical conditions: 1) Classical broad line region (BLR) of size $\sim 4.5 \times 10^{16} \mathrm{~cm}$ is ionized and excited by the central source radiation. The central source brightness variations in 1971-1972 produced gas parameter variations of $10^{9} \mathrm{~cm}^{-3} \geq n_{\mathrm{e}} \geq 10^{8} \mathrm{~cm}^{-3}$ and $2 \times 10^{4} \mathrm{~K} \leq T_{\mathrm{e}} \leq 4 \times 10^{4} \mathrm{~K}$. 2) The profiles of the Balmer lines contain narrow components that have retained their positions (radial velocities) over 25 and 17 years in NGC 3227 and NGC 7469, respectively. These components are supposed to be caused by long-lived gas flows in the nuclei of the galaxies. Observational data acquired by Rubin and Ford allow us to assume that gas in the flows can be explained by models of collision ionization and excitation of gas with self-absorption. It is dense and hot plasma with $n_{\mathrm{e}}=10^{8}-10^{12} \mathrm{~cm}^{-3}$ and $T_{\mathrm{e}}=(1-2.5) \times 10^{4} \mathrm{~K}$. 3) Broad blue bumps of radial velocities between $-4000 \mathrm{~km} \mathrm{~s}^{-1}$ and $-5000 \mathrm{~km} \mathrm{~s}^{-1}$ were detected in the $\mathrm{H} \gamma$ profile during a several day flare twice in NGC 3227 nucleus and once in NGC 7469. One can speculate that the flares are connected with short-lived ejections. The gas in the ejections was ionized and excited by collision processes with $n_{\mathrm{e}} \sim 10^{14} \mathrm{~cm}^{-3}$ and $T_{\mathrm{e}} \sim 25000 \mathrm{~K}$.
\end{abstract}

Key words. shock waves - galaxies: nuclei - black hole physics - line: profiles - line: formation - galaxies: active

\section{Introduction}

In disk accretion models massive black holes $\left(10^{7}-10^{9}\right) M_{\odot}$ is now developed effectively for the central sources of active galactic nuclei (AGNs). But some of AGNs show observational peculiarities, and any model of central sources should account for the corresponding changes. In this paper, we present variations in the emission $\mathrm{H}_{\alpha}, \mathrm{H}_{\beta}$ and $\mathrm{H}_{\gamma}$ line profiles obtained during 5 epochs over 25 years in 1967-1992 for NGC 3227, and during 6 epochs over 17 years in 1972-1989 for NGC 7469.

Since 1971 spectral monitoring of two bright galaxies in the Seyfert list (Seyfert 1943), NGC 3227 and NGC 7469, was carried out with two telescopes. Spectra for NGC 3227 in 1971-1981 and for NGC 7469 in 1971-1972 were acquired by the high speed spectrograph mounted on 2.6-m Shain telescope of Crimean Astrophysical Observatory (Pronik 1971, 1983, 1975a,b). In January 1977, spectra for both nuclei were obtained at prime focus of the 6-m telescope of Special Astrophysical Observatory of USSR. Balmer emission lines with full widths at zero intensity (FWZI) in the range $6000-10000 \mathrm{~km} \mathrm{~s}^{-1}$ showed interesting features. We first derived variability of both the Balmer emission line profiles and Balmer decrement $\mathrm{I}_{\alpha} / \mathrm{I}_{\beta} / \mathrm{I}_{\gamma} / \mathrm{H}_{\delta}$. The physical conditions of the complex gas envelopes in both galaxy nuclei were obtained. Below we analyze the data published by ourthelves in 1975-2006 for the galaxy nuclei of NGC 3227 and NGC 7469 with the data from literature.

\section{Long-term variation of the Balmer lines of the Seyfert 1 nuclei NGC 3227 and NGC 7469}

Light curves of the Seyfert 1 type nuclei have several common characteristics. Intensity of continuum variations in the nucleus of NGC 7469 exhibit ordinary maxima and minima (or simply maxima and minima) within a diaphragm aperture of 5" diameter during of 3-5 years differing by about a factor of 5 (Pronik et al. 1996). A peculiar event, i.e. a deep minimum, accurs once in several decades. Optical brightness of the NGC 7469 nucleus within a diaphragm aperture of $5^{\prime \prime}$ diameter has changed from ordinary minimum to deep minimum by about a factor of 10 .

(Pronik et al. 1996). Pecular events - flares - often occured with duration of about several days both at minima and maxima of the nuclei brightness (Pronik \& Metik 2004).

We compared the shapes of the Balmer line profiles in the spectra of the nuclei of NGC 3227 and NGC 7469 obtained in minimum, in deep minimum, in maximum, and in flare during maximum of the nucleus brightness. All line profiles used both from our data and scanned from literature were calibrated in terms of continuum intensity and the peak of each Balmer emission line. To align the profiles of $\mathrm{H}_{\alpha}, \mathrm{H}_{\beta}$, and $\mathrm{H}_{\gamma}$ lines, we use neighbouring narrow forbidden lines [NII] $\lambda 6583 \AA$, [OIII] $\lambda 4959 \AA$, and [OIII] $\lambda 4363 \AA$, respectively.

\subsection{Compiled data for NGC 3227}

The nucleus of the NGC 3227 galaxy is bright compared with its surroundings. According to Gonzalez Delgado \& Perez (1997) its continuum emission brightness at $5960 \AA$ decreased from the peak to the radius $1^{\prime \prime}$ by a factor of 5 and to radius of 2 .' 5 - by a factor of 20. The flux intensity of the $\mathrm{H}_{\alpha}$ emission decreased by a factor of 30 between distances of $0^{\prime \prime}$ and 2 '.5 from the peak brightness. In this case, the brightness of the nucleus exceed brightness surrounding the nucleus in aperture 1 1. 5 by more than 1 order of magnitude.

Table 1 shows references for the $\mathrm{H} \alpha, \mathrm{H} \beta$, and $\mathrm{H} \gamma$ profiles in NGC 3227 nucleus compiled from the literature. Its columns 
Table 1. The compiled data for the NGC 3227.

\begin{tabular}{ccccc}
\hline \hline Date & Aperture & $\begin{array}{c}\text { Nucleus } \\
\text { brightness }\end{array}$ & Sy type & References \\
\hline 1 & 2 & 3 & 4 & 5 \\
\hline 03.1967 & $?$ & deep minim. & Sy2 & 1 \\
01.1977 & $1^{\prime \prime} 0 \times 1$ '.5 & flare in max. & Sy1 & 2 \\
01.1990 & see Fig. 4 & maximum & Sy1 & 3 \\
01.1992 & $5^{\prime \prime} \times 10^{\prime \prime}$ & flare in max. & Sy1 & 4 \\
03.1992 & $5^{\prime \prime} \times 10^{\prime \prime}$ & maximum & Sy1 & 4 \\
\hline
\end{tabular}

References: 1 - Rubin, Ford (1968); 2 - Pronik, Metik (2004, 2005); 3 - Salamanka et al. (1994); 4 - Winge et al. (1995).

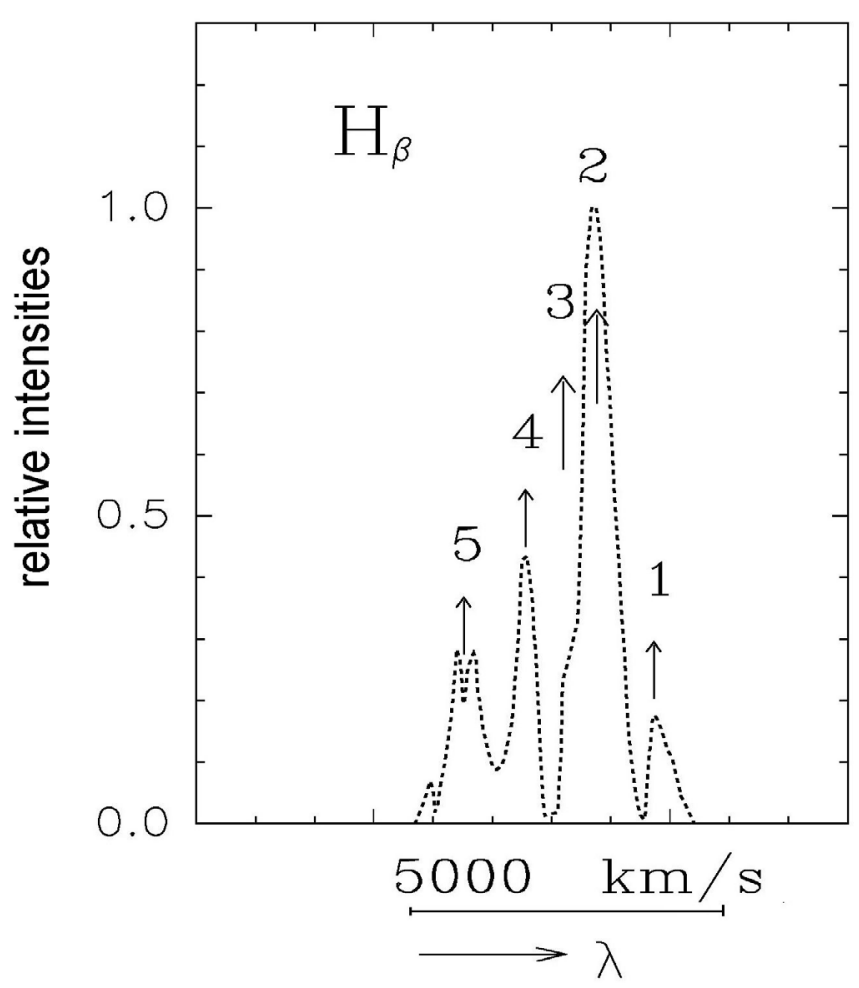

Fig. 1. Multicomponent profile of the $\mathrm{H}_{\beta}$ line for data obtained on March 8, 1967 by Rubin \& Ford (1968) of the spectrum of NGC 3227 nucleus. The arrows show narrow components of the profile 1-5 according to Rubin and Ford.

represent: 1 - date; 2 - aperture of spectra registration; 3 - nucleus brightness; 4 - Seyfert type; 5 - references. Unfortunately, Rubin \& Ford (1968) did not indicate the dimensions of the registration aperture. From the second column of the Table 1, it is clear that the observed nucleus brightness was not influenced by its surroundings.

The data were obtained during 5 epochs between 1967 and 1992. The spectrum of the nucleus varied from Sy 1 type up to Sy 2 type: profiles of the lines changed from having characteristics of both the broad line region (BLR) and narrow-line region (NLR) to those of only the NLR.

Rubin \& Ford (1968) showed that the $\mathrm{H}_{\beta}$ line profile in the spectra of the NGC 3227 nucleus obtained during deep minimum of the brightness in March 1967 was multicomponent. Figure 1 is a copy of Fig. 7, left of the paper by Rubin \& Ford (1968). It shows five individual components in the $\mathrm{H}_{\beta}$ line profile, which we labeled 1-5. All components are sharply defined. The position of the brightest component 2 corresponds to the $\mathrm{H}_{\beta}$ line rest frame. The components 1 and 3-5 are shifted with

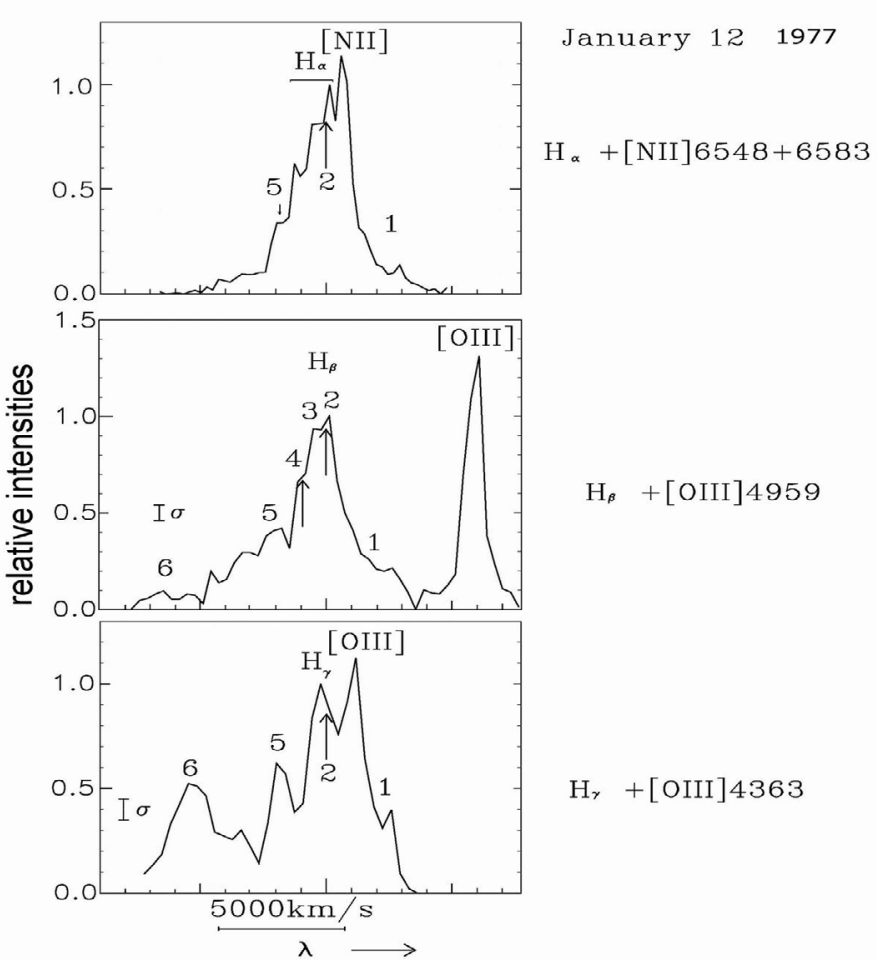

Fig. 2. Profiles of the $\mathrm{H}_{\alpha}, \mathrm{H}_{\beta}$ and $\mathrm{H}_{\gamma}$ emission lines in the spectrum of NGC 3227 on January 12, 1977 are normalized to the continuum and peak intensity by Pronik and Metik (2004). The bars show mean $\mathrm{SD} \sigma$ of the profile. Emission components $1-5$ are the same as in Fig. 1, component 6 - see the text.

respect to the component 2 in velocity units by $\sim+900,-400$, -1000 and $-2000 \mathrm{~km} \mathrm{~s}^{-1}$, respectively. The broad wings of the profile during deep minimum are not observed.

Figure 2 shows the $\mathrm{H}_{\alpha}, \mathrm{H}_{\beta}$, and $\mathrm{H}_{\gamma}$ profiles observed on January 12, 1977 by Pronik and Metik $(2004,2005)$ at the flare during maximum brightness of the NGC 3227 nucleus. Components $1-5$ of the $\mathrm{H}_{\beta}$ line profile are labeled as components $1-5$ in Fig. 1. The blue bump 6 is also present in the $\mathrm{H}_{\gamma}$ and $\mathrm{H}_{\beta}$ profiles. Their widths in the bottom part of the profile are $65 \AA\left(4500 \mathrm{~km} \mathrm{~s}^{-1}\right)$ and $43 \AA\left(2600 \mathrm{~km} \mathrm{~s}^{-1}\right)$, respectively. Their peak intensities are 0.5 and 0.1 of the peak intensities of the $\mathrm{H}_{\gamma}$ and $\mathrm{H}_{\beta}$ profiles respectively.

The shapes of the $\mathrm{H}_{\alpha}, \mathrm{H}_{\beta}$, and $\mathrm{H}_{\gamma}$ profiles are different, which can be due to the high optical depth of the emitting gas.

Figure 3 compares the $\mathrm{H}_{\beta}$ and $\mathrm{H}_{\alpha}$ profiles obtained during both the flare in maximum on January 12, 1977 (Pronik \& Metik 2004, 2005) and flare in deep minimum on March 8, 1967 (Rubin \& Ford 1968). The profiles of the $\mathrm{H}_{\alpha}$ line almost coincide on both dates; they differ only in wings, being broader in 1977. Its broad wings correspond to Sy 1 type. The $\mathrm{H}_{\beta}$ line profiles during the flare in maximum corresponds to Sy 1 type, and during deep minimum it consists only of narrow components and corresponds to Sy 2 type. The position of the 1-5 individual details in the $\mathrm{H}_{\beta}$ profile coincided exactly on both dates.

Figure 4 presents the 2D nucleus spectrum by Salamanca et al. (1994) obtained during the maximum on January 24, 1990. The components $1,(2+3)$ and 4 can be identified separately. It is interesting to note that components 1 and 4 can be detected only in the inner $\sim 1.5^{\prime \prime}$ region and are absent at distances furthers than $0.7^{\prime \prime}$ from the nucleus. 


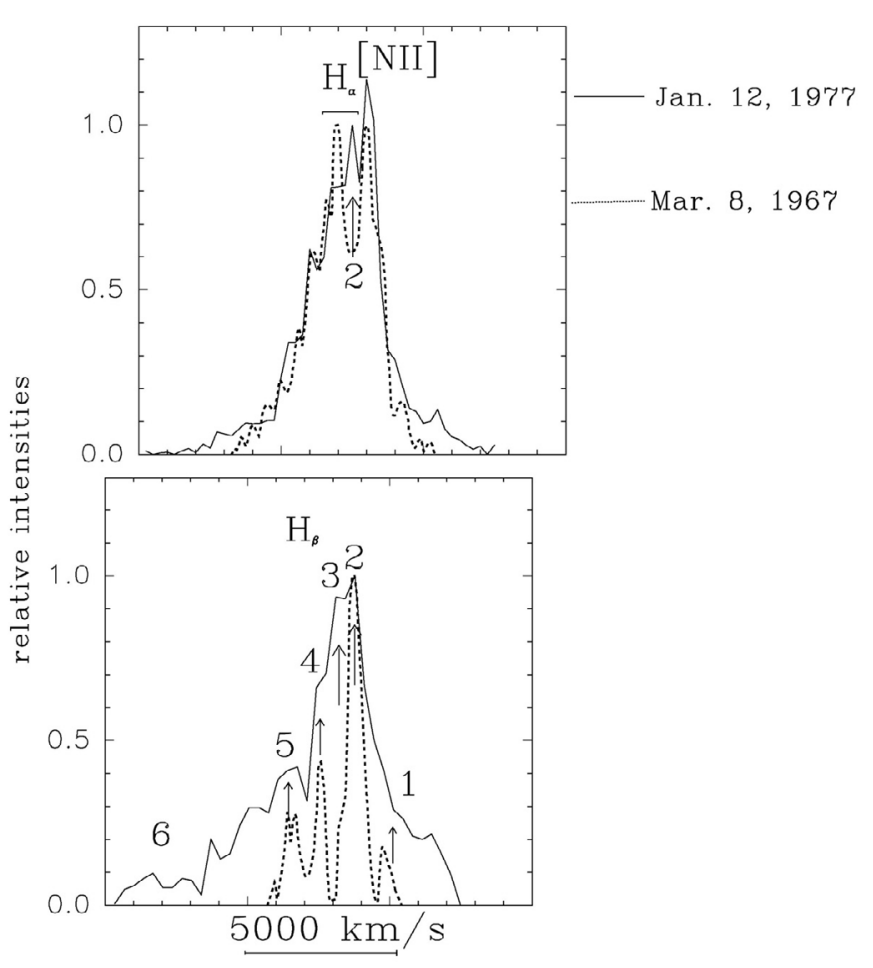

Fig. 3. Comparison of the $\mathrm{H}_{\beta}$ and $\mathrm{H}_{\alpha}$ profiles of the NGC 3227 nucleus on January 12, 1977 by Pronik and Metik (2004, 2005 - solid lines) and on March 8, 1967 by Rubin \& Ford (1968 - dashed lines). The arrows point to the components of the $\mathrm{H}_{\beta}$ line profile common with the components for these two dates.

Figure 5 compares the $\mathrm{H}_{\beta}$ and $\mathrm{H}_{\gamma}$ profiles of the NGC 3227 nucleus obtained during flare in maximum in January 1977 by Pronik and Metik (2004, 2005 - solid lines) and in January 1992 by Winge et al. (1995 - dashed lines). Bright components 1 and 5 of radial velocities +900 and $-2000 \mathrm{~km} \mathrm{~s}^{-1}$ are clearly seen both in the $\mathrm{H}_{\beta}$ and $\mathrm{H}_{\gamma}$ profiles. Blue bump 6 in the $\mathrm{H}_{\gamma}$ profile was more blue-shifted in January 1977 than in January 1992. The difference in their negative radial velocities is about $800 \mathrm{~km} \mathrm{~s}^{-1}$.

Figure 6 compares the $\mathrm{H}_{\beta}$ and $\mathrm{H}_{\gamma}$ line profiles obtained during the flare that occurred at maximum nucleus brightness in January 1977 (Pronik, Metik, 2004, 2005 - solide lines) and at maximum in March 1992 (Winge et al. 1995 - dotted lines). The profiles evolved between flare at maximum in January 1977 and during maximum activity without flare in March 1992. By March 1992, the far blue bump of the $\mathrm{H}_{\beta}$ line had declined and that of the $\mathrm{H}_{\gamma}$ line was absent. The components 1 and 5 in the $\mathrm{H}_{\gamma}$ profile are observable as shoulders, and blue bump 6 of radial velocity $-5000 \mathrm{~km} \mathrm{~s}^{-1}$ is absent.

As a result, two narrow components of the $\mathrm{H}_{\beta}$ and $\mathrm{H}_{\gamma}$ broad line profiles in the NGC 3227 nucleus spectrum have retained their radial velocities -2000 and $+900 \mathrm{~km} \mathrm{~s}^{-1}$ over 25 years. The blue bump of radial velocity $-5000 \mathrm{~km} \mathrm{~s}^{-1}$ was observed during two flares in BLR.

\subsection{Compiled data for NGC 7469}

NGC 7469 is one of the brightest blue galaxies in the first Seyfert list (1943). The size of its unresolved nucleus is less than $1^{\prime \prime}$. CCD observations carried out during the middle phase of the nuclear brightness by Granato et al (1993) indicated that the nuclear continuum inside a $5^{\prime \prime}$ diaphragm is equal to $89 \%, 83 \%$

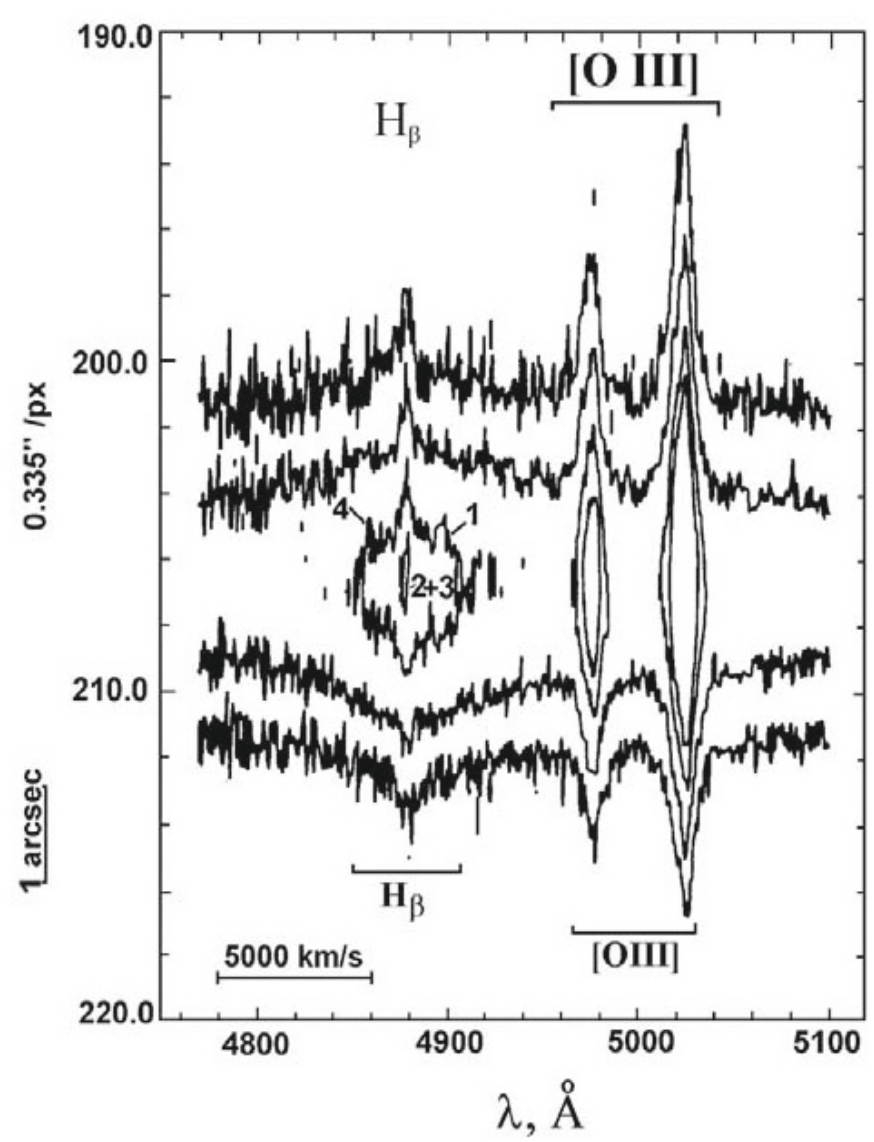

Fig. 4. Isophotal contours of the NGC 3227 nucleus spectrum obtained by Salamanca et al. (1994) on January 24, 1990 at the WHT around the $\mathrm{H}_{\beta}$ and [OIII] lines with the entrance slit width $1.5^{\prime \prime}$. The details of the $\mathrm{H}_{\beta}$ profile are the same as in Fig. 1.

Table 2. Compiled data for NGC 7469.

\begin{tabular}{ccccc}
\hline \hline Date & Aperture & $\begin{array}{c}\text { Nucleus } \\
\text { brightness }\end{array}$ & Sy type & References \\
\hline 1 & 2 & 3 & 4 & 5 \\
\hline 09.1972 & $2^{\prime \prime} \times 10^{\prime \prime}$ & flare in max. & Sy1 & 1 \\
08.1973 & $1^{\prime \prime} 6 \times 22^{\prime \prime} 0$ & maximum & Sy1 & 2 \\
06.1975 & $D=10^{\prime \prime}$ & maximum & Sy1 & 3 \\
10.1975 & $?$ & maximum & Sy1 & 4 \\
01.1977 & $1^{\prime \prime} \times 11^{\prime \prime} 7$ & flare in min. & Sy1 & 5 \\
10.1989 & $1^{\prime \prime \prime} 6 \times 22^{\prime \prime} 0$ & deep minim. & Sy2 & 2 \\
\hline
\end{tabular}

References: 1 - Pronik (1975); 2 - Chuvaev et al. (1990); 3 - de Bruyn, Sargent (1978); 4 - Barbieri et al. (1977); 5 - Pronik et al. (1996, 1997).

and $78 \%$ in $B V R$ bands respectively. According to Mauder et al. (1994), the flux of the continuum at $\lambda 6130 \AA$ decreases by a factor of 7, and both $\mathrm{H}_{\alpha}$ and [OIII] $\lambda 5007 \AA$ fluxes by a factor of 20 inside $0 . " 5$ radius close to the central peak.

Table 2 shows references for the $\mathrm{H}_{\alpha}, \mathrm{H}_{\beta}$, and $\mathrm{H}_{\gamma}$ profiles in the NGC 7469 nucleus spectrum compiled from the literature. Its columns represent: 1 - date; 2 - aperture of spectra registration; 3 - nucleus brightness; 4 - Seyfert type; 5 - references. The data were obtained during 6 epochs between 1972 and 1989. Unfortunately, Barbieri et al. (1977) did not provide the dimensions of their registration aperture. From the second column of Table 2, it is clear that the chracteristics of the variable emission profiles of the nucleus are not influenced by their surroundings. 


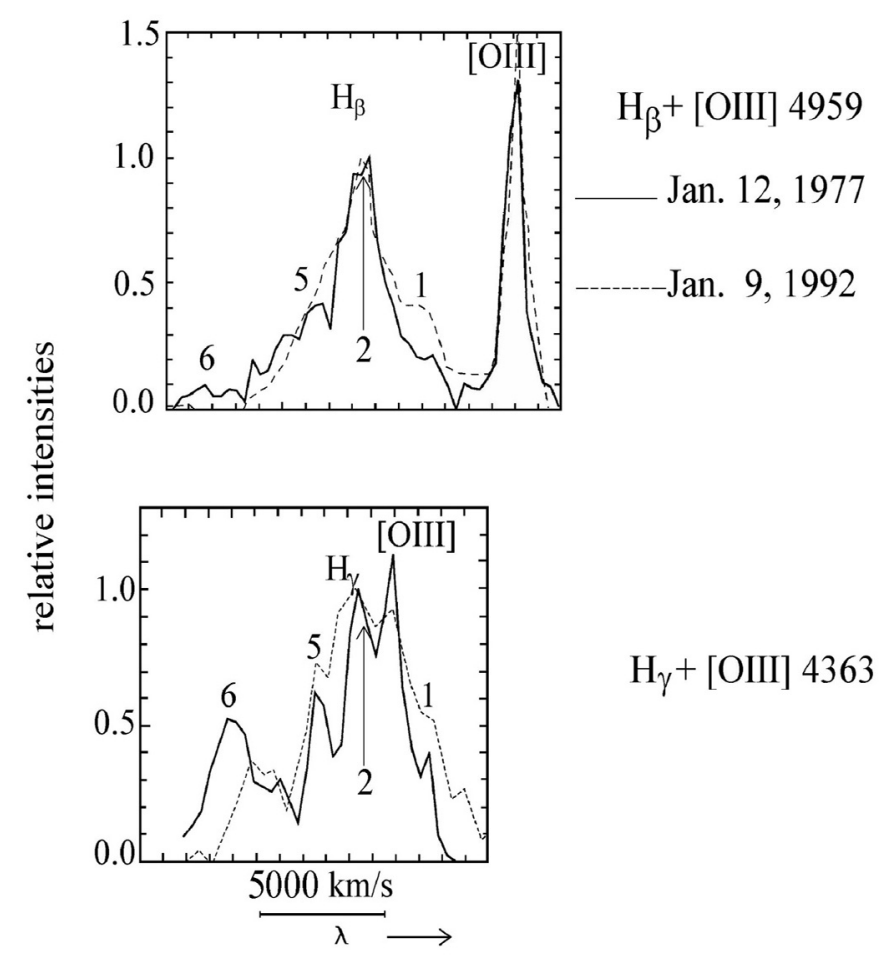

Fig. 5. Comparison of the $\mathrm{H}_{\beta}$ and $\mathrm{H}_{\gamma}$ profiles of the NGC 3227 nucleus during flare in maxima brightness obtained on January 12, 1977 by Pronik and Metik (2004 - solid lines) and on January, 1992 observed by Winge et al. (1995 - dashed lines). The details of the profile are the same as in Figs. 1 and 2.

Pronik (1975) acquired spectroscopic data of the NGC 7469 nucleus during 1971-1972, and found that the profiles of the $\mathrm{H}_{\beta}$, $\mathrm{H}_{\gamma}$, and $\mathrm{H}_{\delta}$ lines contained narrow component that retained their positions throughout the year.

Barbieri et al. (1977) presented the NGC 7469 nucleus spectrum observed in October 25, 1975, during a maximum of nucleus brightness. They stated that "....the data are used in computer program that filters the noise...". Figure 7 shows four individual components in the central part of the $\mathrm{H}_{\beta}$ line profile: "c" - central and "a" with a separation of $+28 \AA$ or $+1700 \mathrm{~km} \mathrm{~s}^{-1}$. One can see also components " $b$ " and "d" separated from the "c" component by $+8 \AA\left(+500 \mathrm{~km} \mathrm{~s}^{-1}\right)$ and by $-6 \AA\left(-350 \mathrm{~km} \mathrm{~s}^{-1}\right)$, respectively.

Figure 8 compares the $\mathrm{H}_{\beta}$ line profile obtained during the flare in maximum of the nucleus brightness on September 18, 1972, by Pronik (1975), with the profiles obtained during maxima on June 7, 1975, by de Bruyn \& Sargent (1978), and in October, 1975 by Barbieri et al. (1977). During the flare in maximum nucleus brightness in September 1972, component "b" was brighter than the central component "c". Components "a" and "d" are discernible as emission or shoulders in the profiles.

Figure 9 compares the $\mathrm{H}_{\beta}$ profile obtained during the maximum of the nucleus brightness on October 25, 1975, by Barbieri et al. (1977), and at minimum nucleus brightness on January 13-15, 1977, by Pronik et al. (1996, 1997). Normalisation of the intensities of the $\mathrm{H}_{\beta}$ profile in Fig. 9 was completed only for two profiles obtained in January 1977. We can not draw a line through the continuum in the spectrum published by Barbieri et al. (1977). Intensities of the components

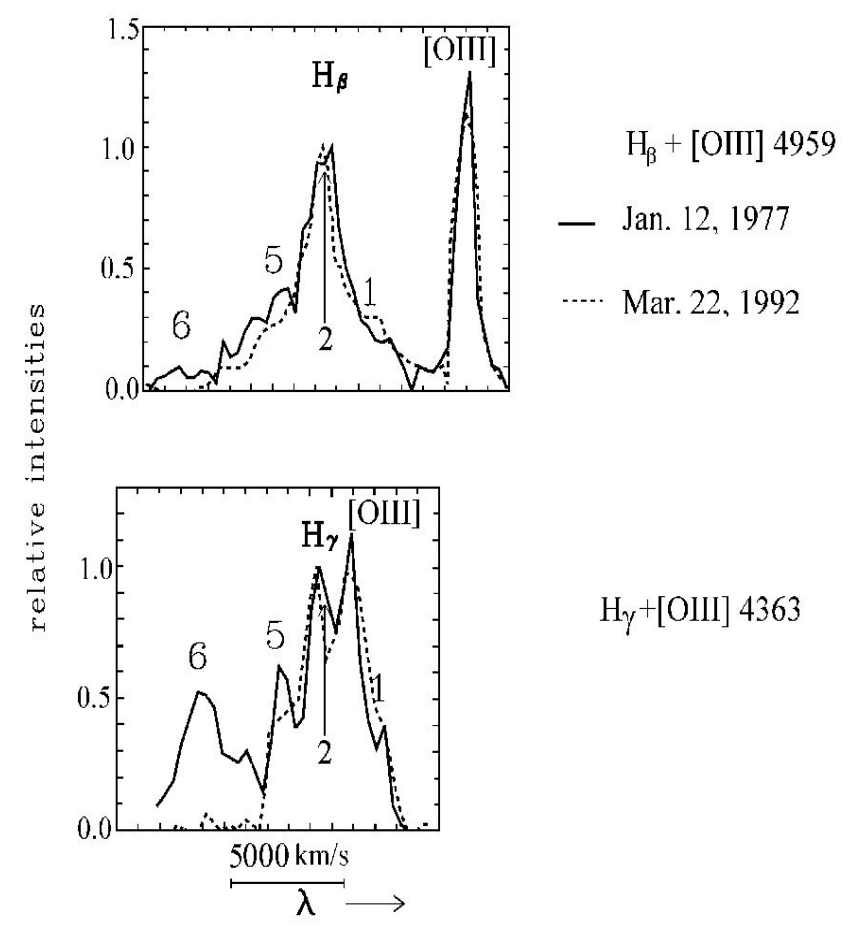

Fig. 6. Comparison of the $\mathrm{H}_{\beta}$ and $\mathrm{H}_{\gamma}$ profiles of the NGC 3227 nucleus obtained during the flare in maximum of the nucleus brightness on January 12, 1977 by Pronik and Metik (2004, 2005 - solid lines) with the profiles of the maximum state in March 1992 observed by Winge et al. (1995 - dashed lines). The details of the profile are the same as in Fig. 5.

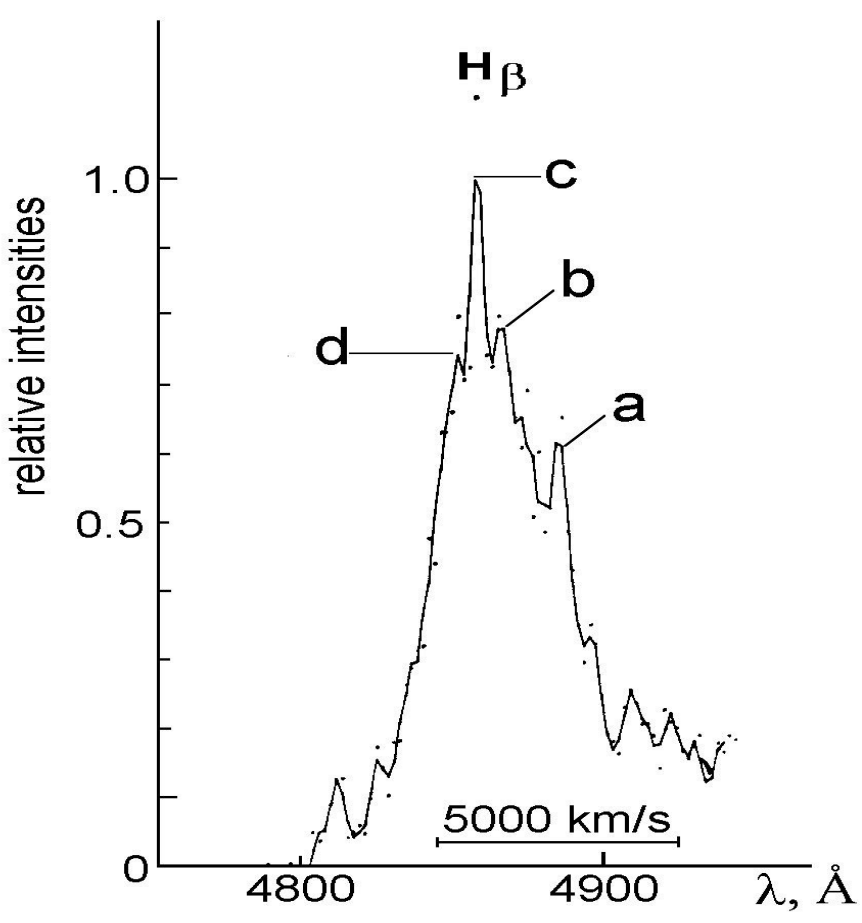

Fig. 7. Profile of the $\mathrm{H}_{\beta}$ line in the spectrum of NGC 7469 nucleus obtained in October 1975 by Barbieri et al. (1977). Four individual components "a-d" are shown. 


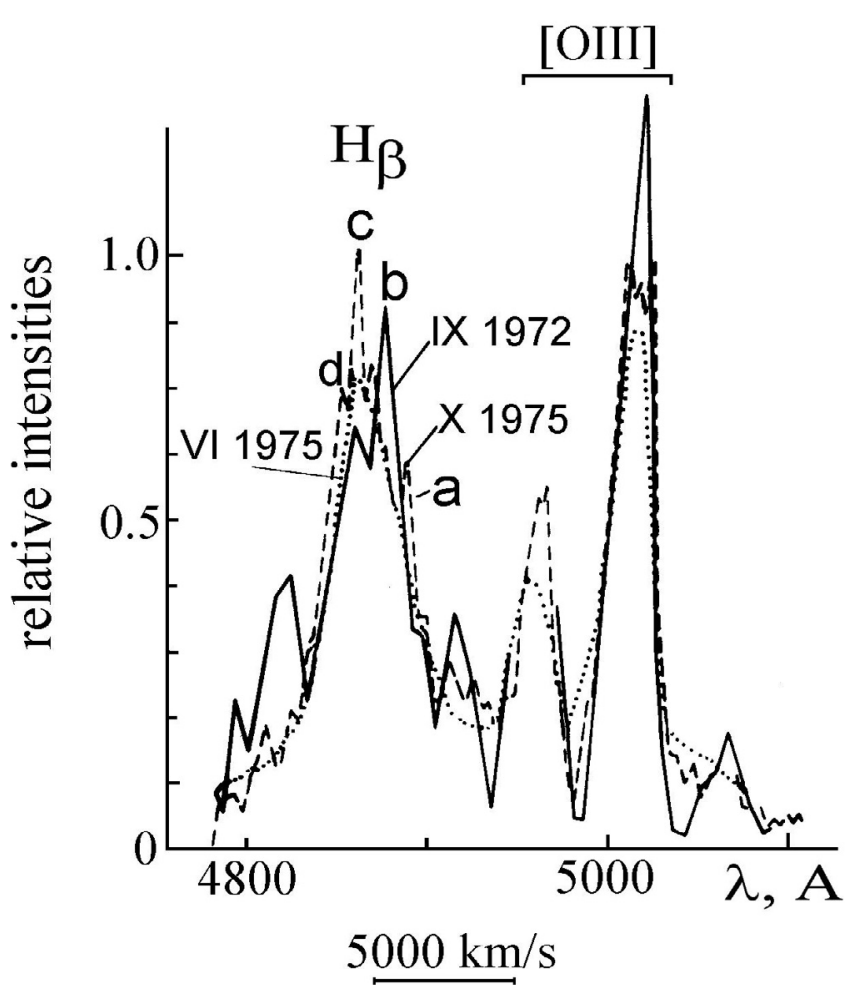

Fig. 8. Comparison of the $\mathrm{H}_{\beta}$ line profiles in the spectrum of NGC 7469 nucleus obtained during the flare at maximum brightness in September 1972 by Pronik (1975), in maxima in June 1975 by de Bruyn \& Sargent (1978), and in October 1975 by Barbieri et al. (1977). The four labeled individual components "(a-d)" are the same as in Fig. 7.

of their profile are given in the arbitrary units. During minimum brightness components "a", "b" and "d" are seen as shoulders.

Figure 10 compares the $\mathrm{H}_{\beta}$ line profiles obtained during a minimum of the nucleus brightness on January $13-15,1977$, by Pronik et al. (1996), and a deep minimum of the nucleus brightness on October 5, 1989, by Chuvaev et al. (1990). Components "a" and "b", observed during deep minimum of the nucleus brightness, on October 5, 1989, are seen as emission, and component " $\mathrm{d}$ " as a shoulder.

We have demonstrated that the profile shape of the $\mathrm{H}_{\beta}$ line obtained during 17 years by different authors in 1972-1989 allows us to argue that narrow components of the profile central part shifted by $+28 \AA\left(+1700 \mathrm{~km} \mathrm{~s}^{-1}\right)$ and $+8 \AA\left(+500 \mathrm{~km} \mathrm{~s}^{-1}\right)$, compare to the central component, which retained their positions (radial velocities) over 17 years during the flare in maximum, in minimum, and in deep minimum of the NGC 7469 nucleus brightness.

\section{Deep minimum as a peculiar event during the AGNs variatiability}

Narrow components of the broad line profiles were best seen during deep minimum of the nuclear brightness for the NGC 3227 nucleus observed in 1967 by Rubin \& Ford (1968), and for the NGC 7469 nucleus by Chuvaev et al. (1990) in 1989. Figures 1 and 10 illustrate how the profiles of the $\mathrm{H}_{\beta}$ line for both nuclei consist of narrow components only and have no broad wings, which are always observed during the high nucleus brightness.

The deep nuclear minimum is a rare and peculiar event that lasts for 2-3 months. According to Chuvaev et al. (1990) and Pronik et al. (1996), the brightness of the NGC 7469 nucleus,

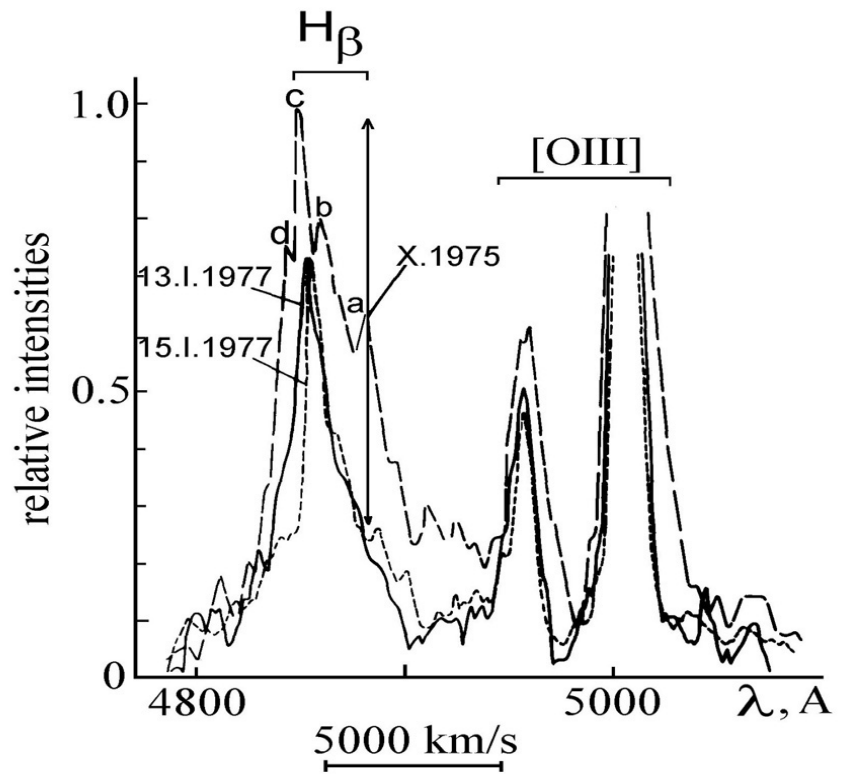

Fig. 9. Comparison of the $\mathrm{H}_{\beta}$ line profiles in the spectrum of NGC 7469 nucleus obtained during minimum brightness on January 13-15, 1977 by Pronik et al. $(1996,1997)$ and maximum in October 1975 by Barbieri et al. (1977). The four individual components are the same as in Fig. 7. The arrow points to the component "a".

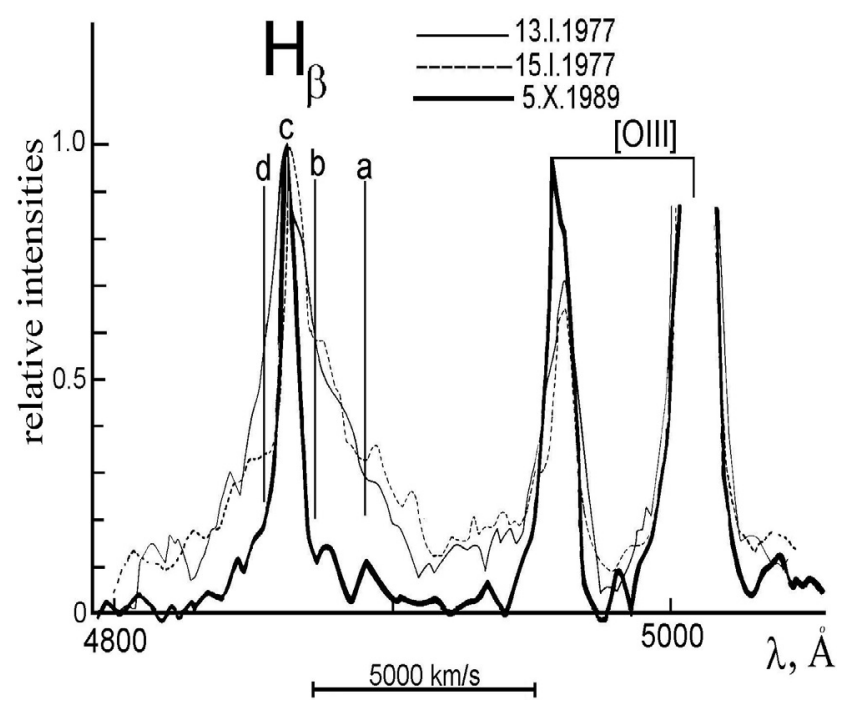

Fig. 10. Comparison of the $\mathrm{H}_{\beta}$ line profiles in the spectrum of NGC 7469 nucleus obtained during minimum brightness on January 13-15, 1977 by Pronik et al. $(1996,1997)$ and deep minimum in October 1989 by Chuvaev et al. (1990). Four individual components are the same as in Fig. 7.

during 50 days of deep minimum in 1989, decreased in diaphragm $5^{\prime \prime}$ by more than a factor of 10 compared with the level of an ordinary minimum. This decreasing led to the changing of the Seyfert type of the nucleus from Sey 1 to Sey 2 .

Figure 11 illustrates high level difference in the general shapes of the $\mathrm{H}_{\alpha}$ and $\mathrm{H}_{\beta}$ profiles during the deep minimum of NGC 3227 nucleus brightness in 1967 according to Rubin \& Ford (1968). The shapes of the line profiles correspond to different Seyfert types: $\mathrm{H}_{\alpha}$ to $\mathrm{Sy} 1$, and $\mathrm{H}_{\beta}$ to $\mathrm{Sy} 2$.

We assume that physical conditions in the gas of narrow components are not the same as in classical BLR. Rubin \& Ford (1968) obtained the ratio $\mathrm{I}_{\alpha} / \mathrm{I}_{\beta}=17$ during a deep minimum of 


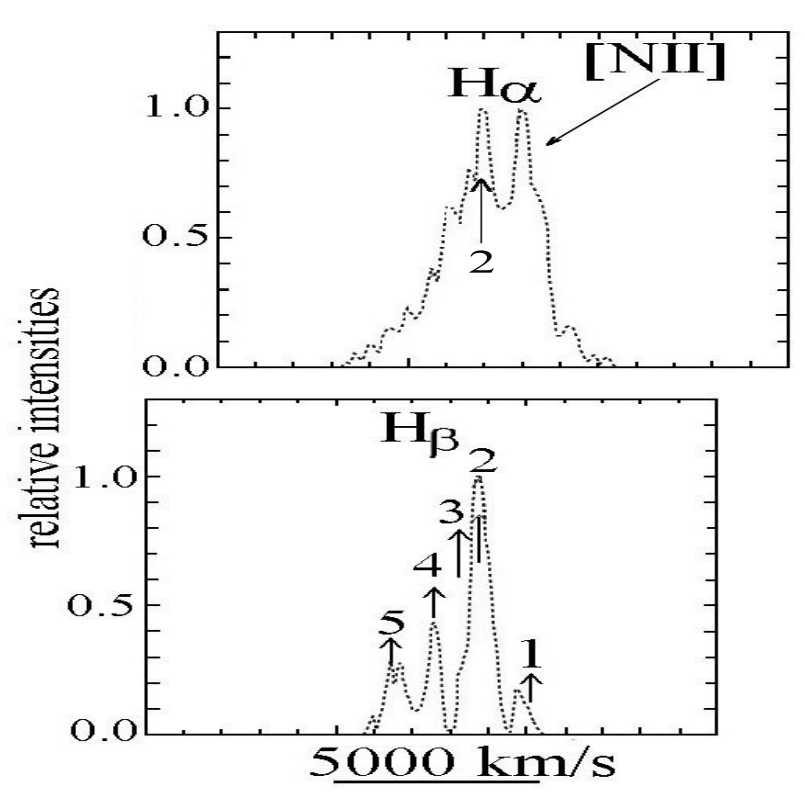

Fig. 11. Profiles of the $\mathrm{H}_{\alpha}$ and $\mathrm{H}_{\beta}$ lines during deep minimum of NGC 3227 nucleus brightness in 1967 according to Rubin \& Ford (1968). Individual components are labeled 1-5 as in Fig. 1.

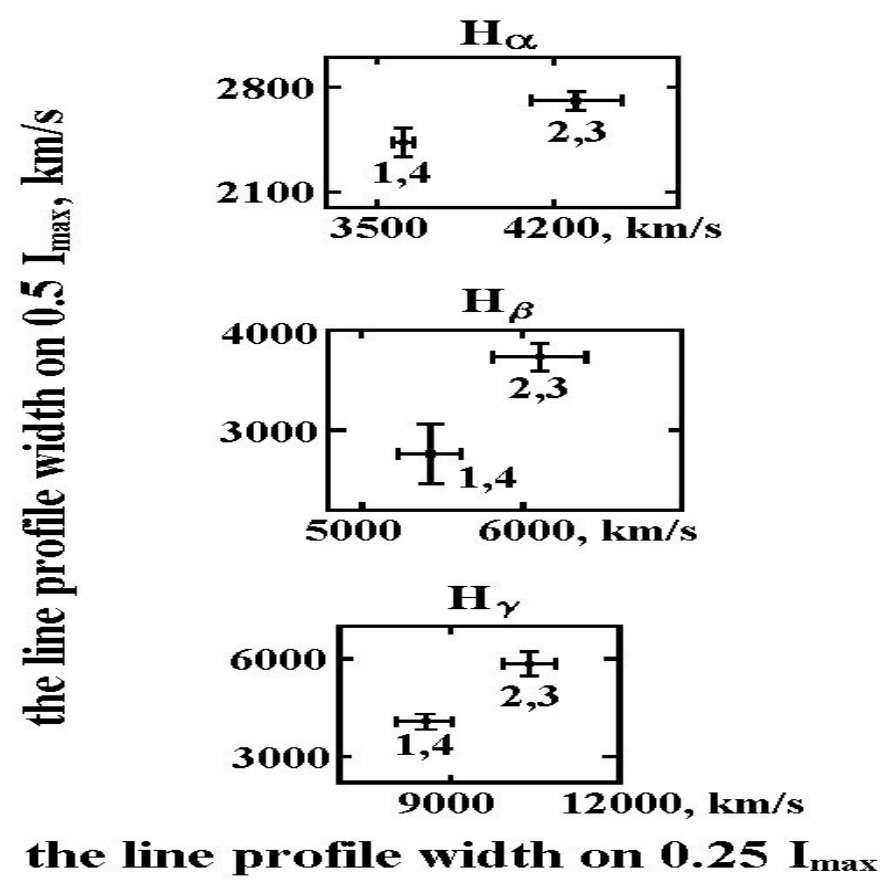

Fig. 12. The comparison of the profile widths of the $\mathrm{H}_{\alpha}, \mathrm{H}_{\beta}$ and $\mathrm{H}_{\gamma}$ lines of the NGC 3227 nucleus on the levels 0.25 and 0.50 of peak intensity observed on January 12-15, 1977 (Metik et al. 2006).

the NGC 3227 nucleus brightness. They showed that data processing and interstellar reddening can not influence the ratio by more than a factor of 1.5 . The ratio $\mathrm{I}_{\alpha} / \mathrm{I}_{\beta}=11$, obtained after all corrections, they attributed to the self-absorption.

The Balmer decrement of the high optical depth gas emission with self-absorption was calculated for the case of radiative ionization and excitation by Boyarchuk (1966), and for the case of collision ionization and excitation by Gershberg and Shnol (1974). Grids of theoretical Balmer decrements calculated by Boyarchuk (1966) and Gershberg and Shnol (1974) for various
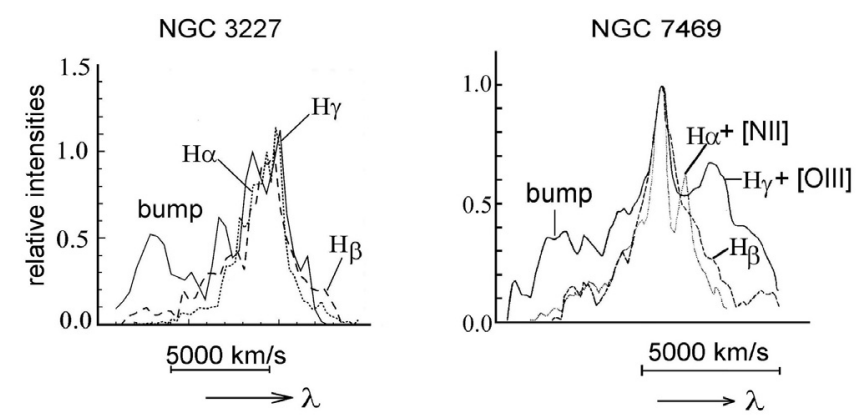

Fig. 13. Profiles of the $\mathrm{H}_{\gamma}, \mathrm{H}_{\beta}$ and $\mathrm{H}_{\alpha}$ lines obtained during 3-day flares for the NGC 3227 and NGC 7469 in January 1977 by Pronik and Metik (2004) and Pronik et al. (1996, 1997), respectively.

electron temperatures and electron concentrations of gas allow us to assume that the Balmer decrement $\mathrm{I}_{\alpha} / \mathrm{I}_{\beta}=11$ can be explained only in the frame work of the model of collision ionization and excitation of gas with self-absorption and $n_{\mathrm{e}}=10^{8}-$ $10^{12} \mathrm{~cm}^{-3}$ and $T_{\mathrm{e}}=(1-2.5) 10^{4} \mathrm{~K}$. It is dense and hot plasma.

Significant differences in the profile shapes of $\mathrm{H}_{\alpha}$ line corresponded to Sy 1 type, and $\mathrm{H}_{\beta}$ line to Sy 2 type, evidence that these lines are emitted by different gas layers that are inhomogeneous in electron density and temperature.

\section{Several day flares in gas of the Broad line region}

Several day flares were revealed by analyzing spectra of both galaxy nuclei. During the flares the $\mathrm{H}_{\alpha}, \mathrm{H}_{\beta}$, and $\mathrm{H}_{\gamma}$ line profiles broadened and intensiry of their narrow components increased compare to the central component peak. In the case of NGC 3227, we observed the flare from its beginning to its end. In Fig. 12, one can see the broadening of the $\mathrm{H}_{\alpha}, \mathrm{H}_{\beta}$, and $\mathrm{H}_{\gamma}$ profiles at the levels 0.25 and 0.50 of the profile peak. Figs. 1-4 correspond to independent days of observations. During the second and third days one can see that the profile widths of the lines were broader than during the first and forth days of observations. The calculated broadening on the level 0.5 profile peak intensity equals $12 \%, 35 \%$, and $44 \%$, respectively. At maxima of the flare, a blue bump at a radial velocity of $-5000 \mathrm{~km} \mathrm{~s}^{-1}$ in the $\mathrm{H}_{\gamma}$ profile was revealed (see Fig. 13, left in the paper by Pronik \& Metik 2004).

In the case of NGC 7469, we observed the maximum broadening and a blue bump in the $\mathrm{H}_{\gamma}$ line profile at the frst day of observations and their declining at the second and third days of observations. The time of decreasing of the $\mathrm{H}_{\gamma}$ profile widths was the same for both NGC 7469 and NGC 3227 nuclei. Taking into account this fact we assume that the duration of the flares in both nuclei was abaut 3 days.

Figure 13 provides the $\mathrm{H}_{\gamma}, \mathrm{H}_{\beta}$, and $\mathrm{H}_{\alpha}$ line profiles obtained during the maxima of 3-day flares for NGC 3227 (Pronik \& Metik 2004) and for NGC 7469 (Pronik et al. 1997). The profiles of the $\mathrm{H}_{\gamma}$ line for both galaxy nucleus spectra contained broad blue bump of radial velocity $-5000 \mathrm{~km} \mathrm{~s}^{-1}$ and $-4000 \mathrm{~km} \mathrm{~s}^{-1}$, respectively.

The blue bump in the $\mathrm{H}_{\gamma}$ profile of NGC 3227 was observed twice in 1977 by Pronik and Metik (2004) and in 1992 by Winge et al. (1995). The blue bump in the $\mathrm{H}_{\gamma}$ profile of NGC 7469 was observed once in 1977 (Pronik et al. 1997). The widths of the blue bumps were $65 \AA$ for NGC 3227 and $45 \AA$ for NGC 7469 , and the peak intensities were about 0.5 and 0.3 of the peak intensity of the $\mathrm{H}_{\gamma}$ profile, respectively. These bumps in the $\mathrm{H}_{\gamma}$ profile for NGC 3227 and NGC 7469 were considered to be the 
results of high radial velocity ejections from the galaxy nuclei during several day flares. The radial velocity of the bump in the case of NGC 3227 varied between 1977 and 1992 by several hundreds $\mathrm{km} \mathrm{s}^{-1}$. This fact implies that the radial velocity of the jets causing blue bumps varies with time. This highlights the difference between the nature of the blue bump and the narrow line components, which retain their radial velocities over several tens of years. The size of the flare region was estimated from its duration in January 1977 to be equal to about 3 light days i.e. $\sim 10^{16} \mathrm{~cm}$.

Emission by the ejections showed an inverse Balmer decrement. The observed Balmer decrement in optical $\mathrm{I}_{\alpha} / \mathrm{I}_{\beta} / \mathrm{I} \gamma$ was used to determine the physical conditions of the gas emitting these emission lines (Osterbrock 1974, Sect. 4). As a rule, the observed Balmer decrement in the optical spectrum of Seyfert nuclei is $\mathrm{I}_{\alpha} \geq \mathrm{I}_{\beta} \geq \mathrm{I}_{\gamma}$. However in the case of the blue bumps, observed during 3-day flares in NGC 3227 and NGC 7469 nuclei, the Balmer decrement is $\mathrm{I}_{\gamma} \geq \mathrm{I}_{\beta}$. The blue bump in the $\mathrm{H}_{\beta}$ line profile was weak, and absent in the $\mathrm{H}_{\alpha}$ line profile. These data demonstrate an inverse Balmer decrement of the blue bump emission.

The comparison of the observed characteristics of the blue bump in the $\mathrm{H}_{\gamma}$ line profiles with the grids of theoretical Balmer dectrements calculated by both Boyarchuk (1966) and Gershberg \& Shnol (1974), for a range of gas electron temperatures and concentrations allows us to be able to assume that the gas emitting of blue bumps in the 3-day flares is optically thick and hot plasma in which collisional ionisation and excitation prevail radiative ionization and excitation. Its $n_{\mathrm{e}} \sim 10^{14} \mathrm{~cm}^{-3}$ and $T_{\mathrm{e}} \sim 25000 \mathrm{~K}$.

\section{Conclusions}

Results of data dicussion on the Balmer line profiles published by us during 1975-2006 for Seyfert galaxies NGC 3227 and NGC 7469 combined with the data compiled from literature are reviewed in this paper. Data for the nucleus of NGC 3227 were acquired over 25 years in 1967-1992 during 5 epochs. Spectral data compiled for the NGC 7469 nucleus were observed over 17 years in 1972-1989 during 6 epochs. Spectra of both nuclei showed significant variability. Both nuclei were of Sy2 type during deep minimum, and of Sy1 type during the maximum and minimum brightness of the nuclei.

The analysis of the Balmer line profiles variability allows us to suppose that their variations are connected with three independent regions of different physical conditions:

1. Classical BLR, which is ionized and excited by the central source radiation. The tight correlation between the brightness variability of broad lines and central source continuum was established. Time variability of hydrogen lines in the NGC 7469 nucleus was estimated to be by about 20 days (Pronik 1975). Salamanka et al. (1994) and Winge et al. (1995) showed that the variable emission of the BLR in $\mathrm{H}_{\alpha}$ and $\mathrm{H}_{\beta}$ lines lags the continuum flux variation at $\lambda 5000-6000 \AA$ of the NGC 3227 nucleus by about 17 days, implying that the spatial scale of BLR is about 17 light days $\left(\sim 4.5 \times 10^{16} \mathrm{~cm}\right)$. Variations in the central source brightness produce variations in the Balmer decrement and average values of $n_{\mathrm{e}}$ and $T_{\mathrm{e}}$. Duding one year in 1971-1972, observations of the variable Balmer decrement $\mathrm{H}_{\alpha} / \mathrm{H}_{\beta} / \mathrm{H}_{\gamma} / \mathrm{H}_{\delta} / \mathrm{H}_{\epsilon}$ of the NGC 7469 nucleus spectrum allowed us to measure the variation in gas parameters in the intervals $10^{9} \mathrm{~cm}^{-3} \geq n_{\mathrm{e}} \geq 10^{8} \mathrm{~cm}^{-3}$ and $2 \times 10^{4} \mathrm{~K}$ $\leq T_{\mathrm{e}} \leq 4 \times 10^{4} \mathrm{~K}$, respectively (Pronik 1975).

2. Flows or jets with radial velocities $-2000 \mathrm{~km} \mathrm{~s}^{-1}$ and $+900 \mathrm{~km} \mathrm{~s}^{-1}$ lasted in the nucleus of NGC 3227 over 25 years, and in NGC 7469 flows with radial velocities $1700 \mathrm{~km} \mathrm{~s}^{-1}$ and $500 \mathrm{~km} \mathrm{~s}^{-1}$ over 17 years. They were observed as narrow components of the Balmer line profiles both at minimum and maximum of the nucleus brightness. The high ratio $\mathrm{I}_{\alpha} / \mathrm{I}_{\beta}$ obtained by Rubin and Ford for the deep minimum of the NGC 3227 nucleus could be explained only in the frame work of the model with collision ionization and excitation of gas with self-absorption. It is a dense, hot plasma with $n_{\mathrm{e}}=10^{8}-10^{12} \mathrm{~cm}^{-3}$ and $T_{\mathrm{e}}=(1-2.5) 10^{4} \mathrm{~K}$.

3. The third region of the Balmer emission lines in AGNs is related to several day flares. They were identifiable as broad blue bumps of radial velocity $-4000 \mathrm{~km} \mathrm{~s}^{-1}$ and $-5000 \mathrm{~km} \mathrm{~s}^{-1}$. The radial velocity of a blue bump in the NGC 3227 flare changed between 1977 and 1992. One can speculate that the flares are connected with short-lived ejections. Emission of the ejections shows an inverse Balmer decrement: $I_{\gamma} \geq I_{\beta}$. The gas of the ejections with $n_{\mathrm{e}} \sim$ $10^{14} \mathrm{~cm}^{-3}, T_{\mathrm{e}} \sim 25000 \mathrm{~K}$ is ionized and excited by collision processes.

The presented data indicate that flows or jets are characteristic not only of long-lived structures of relativistic radio jets, but also of optical nuclear structures. Models of the central sources of AGNs must include flows or jets in their optical structures.

Acknowledgements. I thank Dr. V. I. Pronik and Dr. P. P. Petrov for the helpful discussions, Dr. Yu. S. Efimof, Ya. Poklad, both the scientific and english referees for useful remarks, and both M. A. Smirnova and L. I. Filatova for the technical assistance.

\section{References}

Barbieri, C., di Serego Alighieri, S., \& Sambon, M. 1977, A\&A, 57, 353 Boyarchuk, A. A., Izv., Krymsk. Astrofiz. Obs. 1966, 35, 45

Chuvaev, K. K., Lyuty, V. M., \& Doroshenko, V. T. 1990, Pis'ma Astron Zhurn, 16,867

de Bruyn, A. G., \& Sargent, W. L. W. 1978, AJ, 83, 1257

Gershberg, R. E., \& Shnol, E. E. 1974, Izv. Krymsk. Astrofiz. Obs., 50, 122

Gonzalez Delgado, R. M., \& Perez, E. 1997, MNRAS, 284, 931

Grantano, G. I., Zitelli, N., Bonoli, F., et al. 1993, ApJS, 89, 35

Mauder, W., Weigelt, G., Appenzeller, I., \& Wagner, S. J. 1994, A\&A, 285, 44

Metik, L. P., Pronik, I. I., \& Sharipova, L. M. 2006, Astrofizika, 49, 499

Osterbrock, D. E. 1974, Astrophysica of Gaseous Nebulae, W. H. Freeman and Company, USA, Sect. 4

Pronik, I. 1971, Astron Tsircular, 663, 1

Pronik, I. 1975a, AZh, 52, 481

Pronik, I. 1975b, in Variable stars and stellar evolution, ed. V. Sherwood, \& L. Plaut (Dordrecht-Holland/Boston-USA: D. Reidel Publishing Company), Proc. IAU Symp. 67, 605

Pronik, I. 1983, Izvestiya Crimean Observatiry, 68, 81

Pronik, I. I., \& Metik, L. P. 2004, Astron. Astrophys. Trans., 23, 509

Pronik, I. I., \& Metik, L. P. 2005, Ap\&SS, 299, 245

Pronik, I. I., Metik, L. P., \& Merkulova, N. I. 1996, Ap\&SS, 239, 97

Pronik, I. I., Metik, L. P., \& Merkulova, N. I. 1997, A\&A, 318, 721

Rubin, V., \& Ford, W. 1968, ApJ, 154, 431

Salamanca, J., Alloin, D., Baribaud, T., et al. 1994, A\&A, 282, 742

Seyfert, C. K. 1943, ApJ, 97, 28

Winge, C., Peterson, B. M., Horne, K., Pogge, R. W., et al. 1995, ApJ, 445, 680 